\title{
On the Impact of Correlated Sampling Processes in WSNs with Energy-neutral Operation
}

\author{
Javier Matamoros, Miguel Calvo-Fullana and Carles Antón-Haro \\ Centre Tecnològic de Telecomunicacions de Catalunya (CTTC) \\ Parc Mediterrani de la Tecnologia - 08860 - Castelldefels - Barcelona (Spain) \\ email:first.last@cttc.es
}

\begin{abstract}
In this paper, we consider a communication scenario where multiple EH sensor nodes collect correlated measurements of an underlying random field. The nodes operate in an energyneutral manner (i.e. energy is used as soon as it is harvested) and, hence, the energy-harvesting and sampling processes at the sensor nodes become inter-twined, random and spatially correlated. Under some mild assumptions, we derive the multidimensional linear filter which minimizes the mean square error in the reconstructed measurements at the Fusion Center (FC). We also analyze the impact of correlated and random sampling processes in the resulting distortion and, in order to gain some insight, we particularize the analysis to the case of fully correlated spatial fields and with an asymptotically large number of sensor nodes.
\end{abstract}

\section{INTRODUCTION}

Sensor nodes are usually powered by batteries which can be costly, difficult or even impossible to replace (e.g. when nodes are deployed in remote locations). In recent years, energy harvesting $(\mathrm{EH})$ has emerged as a technology capable of overcoming (or, at least, alleviating) the limitations imposed by non-rechargeable batteries. Specifically, nodes equipped with an energy harvesting device are capable of scavenging e.g., solar, wind, thermal, kinetic energy from the environment [1] and, by doing so, extend their lifetime. Upon being harvested, energy can either be stored in a rechargeable battery or, alternatively, be immediately used for sensing and data transmission.

Energy harvesting has received considerable attention by the wireless communications and information theory communities. For point-to-point scenarios, and under the assumption of known energy and data arrivals (offline optimization), the main focus has been on the derivation of optimal transmission strategies at the sensor node. In [2], the authors study the problem of minimizing the time by which all data packets are transmitted to the destination. A number of authors go one step beyond and investigate the impact of finite energy storage capacity [3] or battery leakage [4]; generalize the analysis to fading channels [5]; or explicitly take into consideration the energy needed for data processing (in addition to data transmission) [6].

This work was partially supported by the Catalan Government under grant SGR2014-1567, the Spanish Government under projects PCIN-2013-027 (ECROPS) and TEC2013-44591-P (INTENSYV), and the European Comission under Grant Agreement 318306 (NEWCOM\#).
In this paper, we consider a communication scenario where multiple EH sensor nodes collect spatially correlated measurements of an underlying random field, and wirelessly transmit them to a remote Fusion Center (FC). In addition, we consider that sensors operate in a strict energy-neutral manner, that is, the nodes will harvest energy and immediately use it for sensing the field and transmitting the measurement to the FC. Under this approach, the sensor node can conduct its expected duties for an infinite amount of time (unless its hardware fails) and possibly be bateryless (e.g., simply equipped with a supercapacitor). Moreover, the energy-harvesting and sampling processes are inter-twined. Specifically, the sampling processes at the sensor nodes become (i) random and, to some extent, (ii) correlated due to the spatial correlation exhibited by the EH process (think e.g., of a number of sensors deployed along a roadside collecting vibrational energy from passing vehicles). In order to properly reconstruct the measurements at the FC, we adopt a multidimensional linear filter which minimizes the quadratic error. Our goal is two-fold, namely, to design such a multi-dimensional filter and to analyze the impact of such correlated and random (EH and) sampling processes in the reconstruction distortion. To that aim, we derive closedform expressions of power spectral density (PSD) of the reconstructed signal. To the best of the authors' knowledge, such an analysis is conducted for the first time in this paper. In order to gain some insight, we particularize the analysis to the case of fully correlated spatial fields and with an asymptotically large number of sensor nodes.

This paper is organized as follows. In Section II the system model is presented. In Section III, the optimal (in mean square error sense) multidimensional linear filter is derived under some mild assumptions. The impact of energy harvesting in the sampling process is evaluated in Section IV and particularized for fully correlated fields in Section V. In Section VI numerical results are provided. And finally, Section VII draws some conclusions of this work.

\section{SySTEM MOdeL}

Consider a sensor network composed of $N$ sensor nodes and one Fusion Center (FC). Each node samples a random field $X_{i}(t) i=1, \ldots, N$, that we model as a jointly Wide Sense Stationary (WSS) zero-mean random processes with individual correlation function given by $\left\{R_{X_{i}}(\tau)\right\}_{i=1}^{N}$. Each 


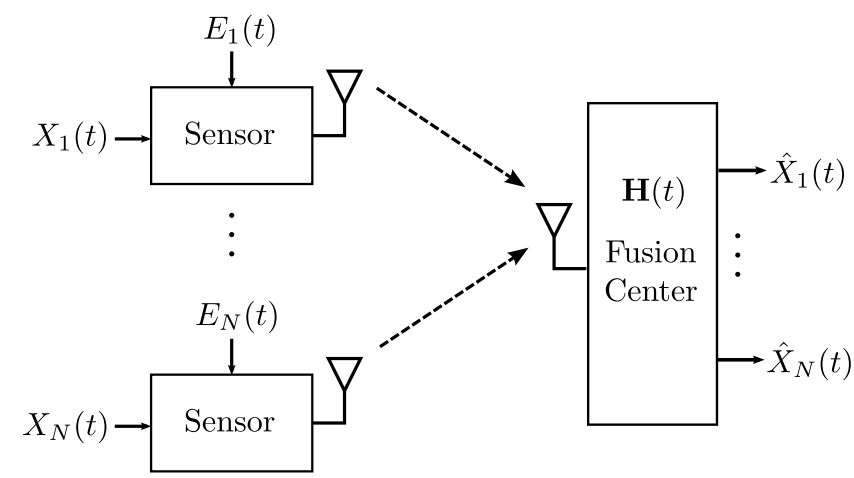

Figure 1. Signal and communication model for a network comprising $N$ energy-harvesting sensors and one fusion center.

sensor nodes is equipped with an energy harvesting device and operates in an energy-neutral manner. That is, upon an energy arrival, the sensor wakes up, samples the field, and reliably transmits the sample to the FC. Accordingly, the sampled signal reads:

$$
\begin{aligned}
X_{s_{i}}(t) & =\left(X_{i}(t)+Z_{i}(t)\right) P_{i}(t) \\
& =\left(X_{i}(t)+Z_{i}(t)\right) \sum_{k=-\infty}^{\infty} \delta\left(t-t_{k}^{(i)}\right)
\end{aligned}
$$

where $P_{i}(t)$ stands for the sampling process at the $i$ th sensor with random sampling times $\left\{t_{k}^{(i)}\right\}$; and $Z_{i}(t)$ denotes band-limited and zero-mean WSS (observation) noise with autocorrelation function $R_{z_{i}}(\tau)$. Due to nodes' energy-neutral operation, the sampling times coincide with energy arrivals. In vector notation, the received signal at the fusion center reads

$$
\mathbf{x}_{s}(t)=(\mathbf{x}(t)+\mathbf{z}(t)) \odot \mathbf{p}(t),
$$

where $\odot$ stands for the Hadamard product, $\mathbf{p}(t)=$ $\left[P_{1}(t), \ldots, P_{N}(t)\right]^{T}$ stands for the sampling processes, and $\mathbf{z}(t)=\left[Z_{1}(t), \ldots, Z_{N}(t)\right]^{T}$ for the observation noise processes. Vectors $\mathbf{x}(t)=\left[X_{1}(t), \ldots, X_{N}(t)\right]^{T}$ and $\mathbf{x}_{s}(t)=$ $\left[X_{s_{1}}(t), \ldots, X_{s_{N}}(t)\right]^{T}$, gather the underlying and received sampled signal; with known correlation matrices $\mathbf{R}_{\mathbf{x x}}(\tau)=$ $\mathbb{E}\left[\mathbf{x}(t) \mathbf{x}^{T}(t+\tau)\right]$ and $\mathbf{R}_{\mathbf{x}_{s} \mathbf{x}_{s}}(\tau)=\mathbb{E}\left[\mathbf{x}_{s}(t) \mathbf{x}_{s}^{T}(t+\tau)\right]$ whose elements are given by

$$
\left[\mathbf{R}_{\mathbf{x}_{s} \mathbf{x}_{s}}(\tau)\right]_{i, j}= \begin{cases}R_{P_{i}}(\tau)\left(R_{X_{i}}(\tau)+R_{Z_{i}}(\tau)\right) & \text { if } i=j \\ R_{P_{i} P_{j}}(\tau) R_{X_{i} X_{j}}(\tau) & \text { if } i \neq j\end{cases}
$$

\section{Computation of the Optimal MULTi-DimEnSIONAL FiLTER}

In order to estimate $\mathbf{x}(t)$, we resort to a multidimensional linear filter defined by the $N \times N$ matrix $\mathbf{H}(t)$,

$$
\mathbf{H}(t)=\left[\begin{array}{cccc}
h_{11}(t) & \ldots & \ldots & h_{1 \mathrm{~N}}(t) \\
\vdots & h_{22}(t) & \ldots & \vdots \\
\vdots & \vdots & \ddots & \vdots \\
h_{N 1}(t) & \ldots & \ldots & h_{N N}(t)
\end{array}\right] .
$$

with $h_{i, j}(t)$ denoting a time-invariant filter. Such a multidimensional approach allows as to leverage on the spatial correlation exhibited by the set of random fields. The $N \times 1$ output of this multidimensional filter reads ${ }^{1}$

$$
\hat{\mathbf{x}}(t)=\int \mathbf{H}(t-u) \mathbf{x}_{s}(u) d u
$$

where, with some abuse of notation, the integrals are computed component-wise. The goal is to find the optimal linear multidimensional filter $\mathbf{H}(t)$ such that the average distortion is minimized. To that end, $\mathbf{H}(t)$ is designed according to the orthogonality principle ${ }^{2}$, namely

$$
\mathbb{E}\left[(\mathbf{x}(t)-\hat{\mathbf{x}}(t)) \mathbf{x}_{s}^{T}(v)\right]=\mathbf{0},
$$

where 0 stands for an all-zero $N \times N$ matrix. From (5), the following must be satisfied:

$$
\mathbb{E}\left[\mathbf{x}(t) \mathbf{x}_{s}^{T}(v)\right]-\mathbb{E}\left[\hat{\mathbf{x}}(t) \mathbf{x}_{s}^{T}(v)\right]=\mathbf{0} .
$$

Note that the first term can be expressed as

$$
\mathbb{E}\left[\mathbf{x}(t) \mathbf{x}_{s}^{T}(v)\right]=\mathbf{R}_{\mathbf{x} \mathbf{x}_{\mathbf{s}}}(t-v)=\mathbf{R}_{\mathbf{x x}_{\mathbf{s}}}(\tau),
$$

where we have used the change of variables $\tau=t-v$. The second term is given by

$$
\begin{aligned}
\mathbb{E}\left[\hat{\mathbf{x}}(t) \mathbf{x}_{s}^{T}(u)\right] & =\mathbb{E}\left[\int \mathbf{H}(t-u) \mathbf{x}_{s}(u) d u \mathbf{x}_{s}^{T}(v)\right] \\
& =\int \mathbf{H}(t-u) \mathbf{R}_{\mathbf{x}_{s} \mathbf{x}_{s}}(u-v) d u . \\
& =\int \mathbf{H}(w) \mathbf{R}_{\mathbf{x}_{s} \mathbf{x}_{s}}(\tau-w) d w
\end{aligned}
$$

where, we have applied the change of variables $w=t-u$ and, again, $\tau=t-v$. The individual matrix entries of the equation above are given by

$$
\left[\int \mathbf{H}(w) \mathbf{R}_{\mathbf{x}_{s} \mathbf{x}_{s}}(\tau-w) d w\right]_{k, l}=\sum_{i=1}^{N} h_{k l}(\tau) * R_{x_{s_{k}} x_{s_{l}}}(\tau) .
$$

where $*$ denotes convolution. Hence, from (5), we have

$$
\mathbf{R}_{\mathbf{x x}_{s}}(\tau)=\int \mathbf{H}(w) \mathbf{R}_{\mathbf{x}_{s} \mathbf{x}_{s}}(\tau-w) d w .
$$

By defining $\mathbf{S}_{\mathbf{x} \mathbf{x}_{s}}(f)=\mathcal{F}\left\{\mathbf{R}_{\mathbf{x} \mathbf{x}_{s}}(\tau)\right\}, \mathbf{S}_{\mathbf{H}}(f)=\mathcal{F}\{\mathbf{H}(\tau)\}$ and $\mathbf{S}_{\mathbf{x}_{s} \mathbf{x}_{s}}(f)=\mathcal{F}\left\{\mathbf{R}_{\mathbf{x}_{s} \mathbf{x}_{s}}(\tau)\right\}$, with $\mathcal{F}\{\cdot\}$ standing for the component-wise Fourier transform, equation (6) becomes

$$
\mathbf{S}_{\mathbf{x} \mathbf{x}_{s}}(f)=\mathbf{S}_{\mathbf{H}}(f) \mathbf{S}_{\mathbf{x}_{s} \mathbf{x}_{s}}(f) .
$$

Therefore, the spectral matrix of the best linear multidimensional filter can be computed as follows:

$$
\mathbf{S}_{\mathbf{H}}(f)=\mathbf{S}_{\mathbf{x} \mathbf{x}_{s}}(f) \mathbf{S}_{\mathbf{x}_{s} \mathbf{x}_{s}}^{-1}(f)
$$

and, finally, the optimal linear multidimensional filter yields:

$$
\mathbf{H}(t)=\mathcal{F}^{-1}\left\{\mathbf{S}_{\mathbf{H}}(f)\right\}
$$

with $\mathcal{F}^{-1}\{\cdot\}$ standing for the component-wise inverse Fourier transform.

\footnotetext{
${ }^{1}$ Integration intervals are $-\infty$ to $\infty$, unless otherwise stated.

${ }^{2}$ In other words, the error in the estimate $\mathbf{w}(t)=\mathbf{x}(t)-\hat{\mathbf{x}}(t)$ is forced to be orthogonal to all sensor samples $\mathbf{x}_{s}(u)$. For more information the interested reader is referred to [7]
} 


\section{A. Distortion}

First, from the orthogonality principle (5), we have that

$$
\mathbf{x}(t)=\hat{\mathbf{x}}(t)+\mathbf{w}(t)
$$

with $\mathbf{w}(t)$ being orthogonal to $\hat{\mathbf{x}}(t)$. Hence, by considering the Mean Square Error (MSE) as the distortion metric, the distortion in the estimate of $X_{i}(t)$ can be readily computed as follows:

$$
\begin{aligned}
\mathrm{MSE} & =\mathbb{E}\left[\left(X_{i}(t)-\hat{X}_{i}(t)\right)^{2}\right] \\
& =R_{X_{i}}(0)-R_{\hat{X}_{i}}(0) \\
& =\int S_{X_{i}}(f)-S_{\hat{X}_{i}}(f) d f
\end{aligned}
$$

where $S_{\hat{X}_{i}}(f)$ denotes the Power Spectral Density (PSD) of $\hat{X}_{i}(f)$ and is the $i$-th diagonal element of the following PSD matrix:

$$
\mathbf{S}_{\hat{\mathbf{x}}}(f)=\mathbf{S}_{\mathbf{x x}_{s}}(f) \mathbf{S}_{\mathbf{x}_{s} \mathbf{x}_{s}}^{-1}(f) \mathbf{S}_{\mathbf{x} \mathbf{x}_{s}}^{H}(f)
$$

\section{INTERPLAY OF ENERGY HARVESTING AND SAMPLING PROCESSES}

As in [2]-[5], [8], the energy harvesting processes are modeled as counting processes, namely

$$
E_{i}(t)=\sum_{k=1}^{\infty} \varepsilon_{k}^{(i)} u\left(t-t_{k}^{(i)}\right),
$$

where $u(\cdot)$ stands for the Heaviside function, $\left\{t_{k}^{(i)}\right\}$ denotes the set of random energy arrival times and $\left\{\varepsilon_{k}\right\}$ their corresponding energy amounts. As in [9], we realistically consider identical amounts of harvested energy that is, $\varepsilon_{k}=\varepsilon \forall k$. And, further, that $\varepsilon$ Joules suffices to acquire, process and reliably transmit a sensor sample to the FC. As a result, the sampling times coincide with the energy arrival times (see Figure 2). Thus, the point (sampling) process $P_{i}(t)$ can be expressed as follows:

$$
P_{i}(t)=\frac{d}{d t} E_{i}(t)
$$

where, without loss of generality, we have considered $\varepsilon=1$.

The aim of this section is to assess the impact of the correlation in the energy harvesting processes on the PSD of the sampling processes, which are needed to compute $\mathbf{H}(t)$. For mathematical tractability and motivated by the recent literature on energy harvesting [10]-[12], we model $\left\{E_{i}(t)\right\}$ as a set of Poisson counting processes of intensity rates $\left\{\check{\lambda}_{i}\right\}$. Further, we assume that the energy harvesting processes are spatially correlated ${ }^{3}$ and model such spatial correlation as follows:

$$
E_{i}(t)=E_{c}(t)+S_{i}(t)
$$

where both processes $E_{c}(t)$ and $S_{i}(t)$ stand for Poisson counting processes of intensity rates $\lambda_{c}$ and $\lambda_{i}$, respectively.

\footnotetext{
${ }^{3}$ This models situations where sensors are located close to the same energy harvesting source.
}

Essentially, $E_{c}(t)$ models the common part of the energy arrivals and $S_{i}(t)$ accounts for the sensor-specific (i.e. innovation) part. As shown in Figure 3, this entails some correaltion in the resulting sampling patterns.

The autocorrelation function of $E_{i}(t)$ reads

$$
\begin{aligned}
R_{E_{i}}\left(t_{1}, t_{2}\right) & =\mathbb{E}\left[E_{i}\left(t_{1}\right) E_{i}\left(t_{2}\right)\right] \\
& =\left(\lambda_{c}+\lambda_{i}\right) \min \left(t_{1}, t_{2}\right)+\left(\lambda_{c}+\lambda_{i}\right)^{2} t_{1} t_{2} .
\end{aligned}
$$

Now, since the processes are mean square differentiable, the derivative and expectation operators can be exchanged [7], thus yielding

$$
\begin{aligned}
R_{P_{i}}\left(t_{1}, t_{2}\right) & =\frac{\partial}{\partial t_{1} \partial t_{2}} R_{E_{i}}\left(t_{1}, t_{2}\right) \\
& =\left(\lambda_{c}+\lambda_{i}\right) \delta(\tau)+\left(\lambda_{c}+\lambda_{i}\right)^{2}
\end{aligned}
$$

with $\tau=t_{2}-t_{1}$. The cross-correlation function is given by

$$
\begin{aligned}
R_{E_{i} E_{j}}\left(t_{1}, t_{2}\right)= & \mathbb{E}\left[E_{i}\left(t_{1}\right) E_{j}\left(t_{2}\right)\right] \\
= & \mathbb{E}\left[E_{c}\left(t_{1}\right) E_{c}\left(t_{2}\right)\right]+\mathbb{E}\left[E_{c}\left(t_{1}\right) S_{j}\left(t_{2}\right)\right] \\
& +\mathbb{E}\left[S_{i}\left(t_{1}\right) E_{c}\left(t_{2}\right)\right]+\mathbb{E}\left[S_{i}\left(t_{1}\right) S_{j}\left(t_{2}\right)\right],
\end{aligned}
$$

where

$$
\begin{aligned}
& \mathbb{E}\left[E_{c}\left(t_{1}\right) E_{c}\left(t_{2}\right)\right]=\lambda_{c} \min \left(t_{1}, t_{2}\right)+\lambda_{c}^{2} t_{1} t_{2}, \\
& \mathbb{E}\left[E_{c}\left(t_{1}\right) S_{j}\left(t_{2}\right)\right]=\lambda_{c} \lambda_{j} t_{1} t_{2}, \\
& \mathbb{E}\left[S_{i}\left(t_{1}\right) E_{c}\left(t_{2}\right)\right]=\lambda_{i} \lambda_{c} t_{1} t_{2} \\
& \mathbb{E}\left[S_{i}\left(t_{1}\right) S_{j}\left(t_{2}\right)\right]=\lambda_{i} \lambda_{j} t_{1} t_{2}
\end{aligned}
$$

Again, by exchanging the derivative and expectation operators, it yields

$$
\begin{aligned}
R_{P_{i} P_{j}}\left(t_{1}, t_{2}\right) & =\frac{\partial}{\partial t_{1} \partial t_{2}} R_{E_{i} E_{j}}\left(t_{1}, t_{2}\right) \\
& =\lambda_{c} \delta(\tau)+\lambda_{c}^{2}+\lambda_{c}\left(\lambda_{j}+\lambda_{i}\right)+\lambda_{i} \lambda_{j} .
\end{aligned}
$$

Therefore, the power spectral density of the sampling processes reads:

$S_{P_{i} P_{j}}(f)= \begin{cases}\left(\lambda_{c}+\lambda_{i}\right)+\left(\lambda_{c}+\lambda_{i}\right)^{2} \delta(f) & \text { if } i=j \\ \lambda_{c}+\left(\lambda_{c}^{2}+\lambda_{c}\left(\lambda_{j}+\lambda_{i}\right)+\lambda_{i} \lambda_{j}\right) \delta(f) & \text { if } i \neq j\end{cases}$

Finally, from (3), the PSD of the sampled signal yields:

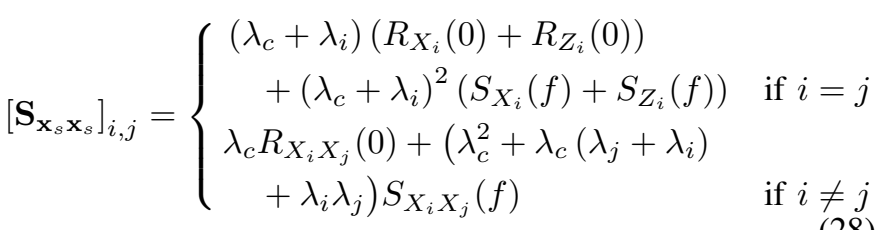

and

$$
\left[\mathbf{S}_{\mathbf{x} \mathbf{x}_{s}}\right]_{i, j}=\left(\lambda_{c}+\lambda_{j}\right) S_{X_{i} X_{j}}(f) .
$$




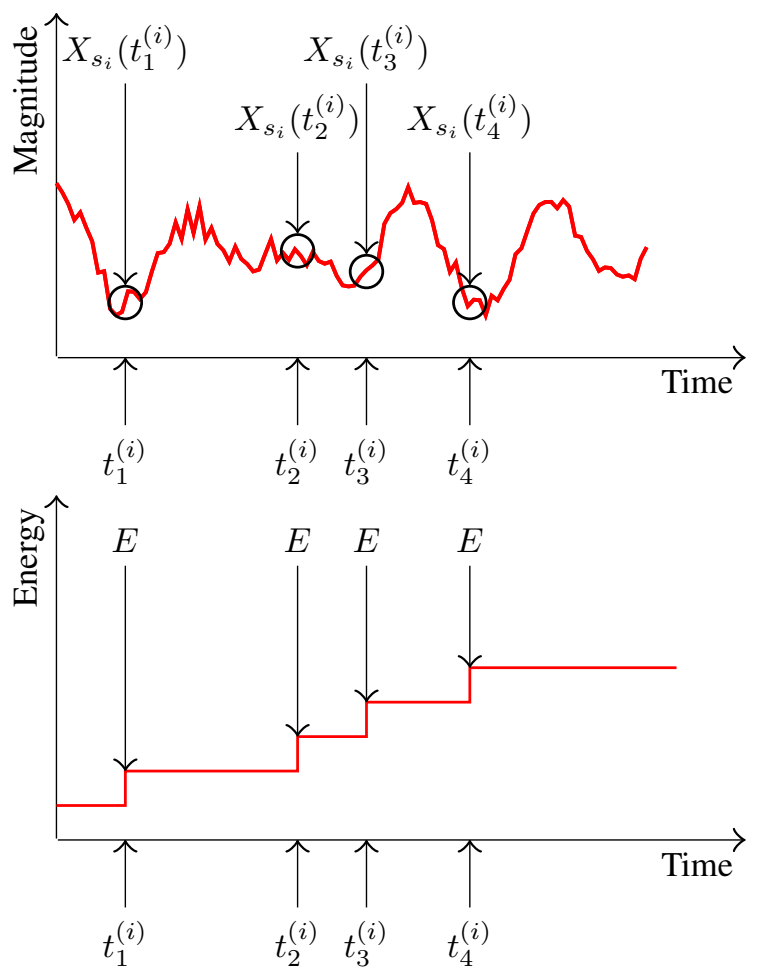

Figure 2. Sampled random field (top) and relation between the energy harvesting and sampling processes (bottom).

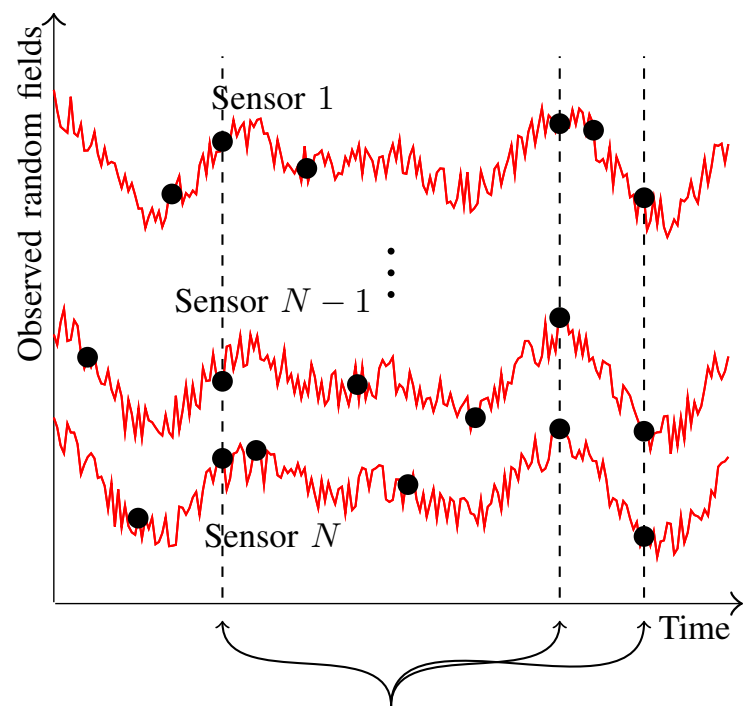

Common sampling times

Figure 3. Graphical representation of the correlated sampling processes at the sensor nodes.

\section{Particular Case: Fully Correlated Fields}

To analyze the impact of correlated sampling processes on the distortion, we consider a scenario where sensor nodes observe the same underlying phenomenon, namely $X_{i}(t)=X(t)$ for $i=1, \ldots, N$. Particularing (4), the estimate of the (single) spatial field is given by:

$$
\hat{X}(t)=\int \mathbf{h}^{T}(t-u) \mathbf{x}_{s}(u) d u
$$

where $\mathbf{h}(t)=\left[h_{1}(t), \ldots, h_{N}(t)\right]^{T}$. Along the lines of Section III, we have that

$$
\begin{aligned}
\mathbf{s}_{\mathbf{h}}(f)^{T} & =\mathcal{F}\left\{\mathbf{h}^{T}(t)\right\} \\
& =\mathbf{s}_{X \mathbf{x}_{s}}^{T}(f) \mathbf{S}_{\mathbf{x}_{s} \mathbf{x}_{s}}^{-1}(f)
\end{aligned}
$$

and

$$
\begin{aligned}
\mathbf{s}_{X \mathbf{x}_{s}}(f) & =\mathcal{F}\left\{\mathbb{E}\left[X(t) \mathbf{x}_{s}(t+\tau)\right]\right\} \\
& =\left(\lambda_{c}+\lambda_{i}\right) S_{X}(f) \mathbf{1}_{N},
\end{aligned}
$$

with $\mathbf{1}_{N}$ standing for the ones vector of length $N$. In order to obtain a simple yet informative expression of the MSE, we assume that the sensor-specific parts of the energy harvesting processes are statistically identical, that is, $\lambda_{i}=\lambda$ and, further, $R_{Z_{i}}(\tau)=R_{Z}(\tau)$. After some algebra, one concludes that:

$$
\begin{aligned}
\mathrm{MSE} & =R_{X}(0)-\int S_{\hat{X}}(f) d f \\
& =R_{X}(0)-\int \frac{N G_{1}(f)}{G_{2}(f)+N G_{3}(f)} d f
\end{aligned}
$$

with

$$
\begin{aligned}
G_{1}(f)= & \left(\lambda_{c}+\lambda\right)^{2}\left|S_{X}(f)\right|^{2}, \\
G_{2}(f)= & \lambda\left(R_{X}(0)+R_{Z}(0)\right)+\lambda_{c} R_{Z}(0) \\
& +\left(\lambda+\lambda_{c}\right)^{2} S_{Z}(f), \\
G_{3}(f)= & \lambda_{c} R_{X}(0)+\left(\lambda+\lambda_{c}\right)^{2} S_{X}(f) .
\end{aligned}
$$

Interestingly, the second term in (35) is a monotonically increasing function in $N$ and, thus, the MSE will decrease monotonically in $N$. This means that the impact of the network size is twofold. First, for an increasing number of sensors, the FC can smooth the noise better, this yielding a lower MSE. Second, increasing the number of sensors also increases the effective sampling rate of the phenomenon as long as the harvesting processes have independent components (see next subsection).

\section{A. Asymptotic Regime}

Taking the limit of the MSE in (38) with respect to $N$, we have that

$$
\begin{aligned}
\lim _{N \rightarrow \infty} \mathrm{MSE} & =R_{X}(0)-\lim _{N \rightarrow \infty} \int \frac{N G_{1}(f)}{G_{2}(f)+N G_{3}(f)} d f \\
& =R_{X}(0)-\int \lim _{N \rightarrow \infty} \frac{N G_{1}(f)}{G_{2}(f)+N G_{3}(f)} d f \\
& =R_{X}(0)-\int \frac{G_{1}(f)}{G_{3}(f)} d f \\
& =R_{X}(0)-\int \frac{\left(\lambda+\lambda_{c}\right)^{2}\left|S_{X}(f)\right|^{2}}{\lambda_{c} R_{X}(0)+\left(\lambda+\lambda_{c}\right)^{2} S_{X}(f)} d f,
\end{aligned}
$$

where (40) follows from the Lebesgue's dominated convergence theorem. First, from (42), one observes that the estimate 
is consistent since the noise term vanishes for large $N$. Besides, for independent harvesting processes, i.e. $\lambda_{c}=0$, the distortion turns out to be zero. This follows from the fact that the sum of $N$ independent Poisson counting processes of average arrival rate $\lambda$ is equivalent to have a single Poisson counting processes of average rate given by $N \lambda$. Hence, for large $N$, the equivalent average sampling rate tends to infinity. For correlated sampling processes $\left(\lambda_{c}>0\right)$ there exists some sampling noise (with power $\lambda_{c} R_{X}(0)$ ) that has a negative impact on the resulting MSE. From (42), note that this effect can only be alleviated by letting $\lambda_{c}$ grow to infinity (since the rest of the terms in the integral grow with $\lambda_{c}^{2}$ ).

\section{Numerical RESUlts}

For a numerical comparison, we consider the scenario of Section V where sensors observe the same random field. In particular, we consider the estimation of a Markov Gaussian process with spectral density given by

$$
S_{X}(f)=\frac{2 \sigma_{x}^{2} \beta}{4 \pi^{2} f^{2}+\beta^{2}},
$$

where $\sigma_{x}^{2}$ and $\beta$ are the parameters modeling the energy and variability of the phenomenon. Regarding the observation noise, we consider band-limitted Gaussian noise with spectral density given by:

$$
S_{Z}(f)=\left\{\begin{array}{cc}
\varepsilon_{z} & \text { if }|f|<f_{\max } \\
0 & \text { otherwise. }
\end{array}\right.
$$

Figure 4 reveals that distortion is a decreasing function in the number of sensor nodes. As discussed in Section V-A, for independent harvesting processes the MSE tends to 0 for large $N$. This is illustrated in Figure 5, where the PSD of the underlying process $X(t)$, given by (43), is compared with the PSDs of the resulting reconstructions. In this example, with $N=1000$ and independent harvesting processes $\left(\lambda_{c}=0\right)$, the PSDs of the actual and the reconstructed processes are virtually identical. For correlated harvesting processes, on the contrary and as shown in Figure 4, the MSE saturates beyond.

More interestingly, as shown in Figure 4 , the required average rate of harvested energy, given by $N \cdot\left(\lambda+\lambda_{c}\right)$, to attain a prescribed value of MSE, can be substantially different depending on the statistical properties of the energy harvesting processes. For instance, for independent harvesting processes $\left(\lambda_{i}=0.1\right.$ and $\left.\lambda_{c}=0\right)$, the required energy rate to achieve a MSE $\approx 0.04$ is 5 , whereas it turns out to be 30 for correlated energy harvesting processes $\left(\lambda_{i}=0.5\right.$ and $\left.\lambda_{c}=0.1\right)$.

\section{CONCLUSIONS}

In this paper, we have considered the reconstruction of correlated random fields with wireless sensor networks that operate in an energy-neutral manner. First, under the assumption of WSS random sampling processes, we have proposed a multidimensional linear filter that exploits the correlation between the sensor observations. Next, we have computed the power spectral density of the sampling processes due to the energy neutral operation of the nodes. For this case, we

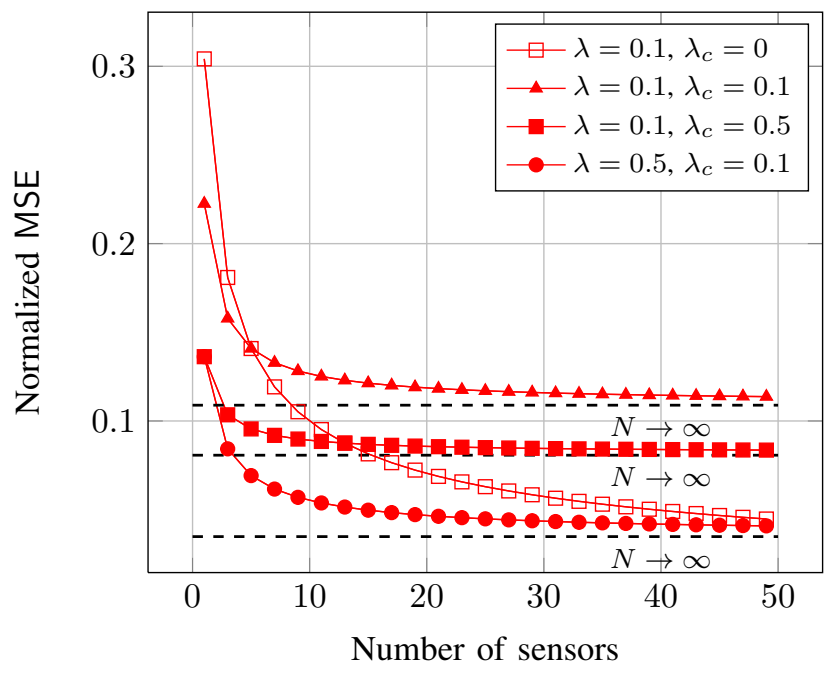

Figure 4. Normalized MSE vs number of sensors for different (average) energy harvesting rates values of $\lambda$ and $\lambda_{c}$.

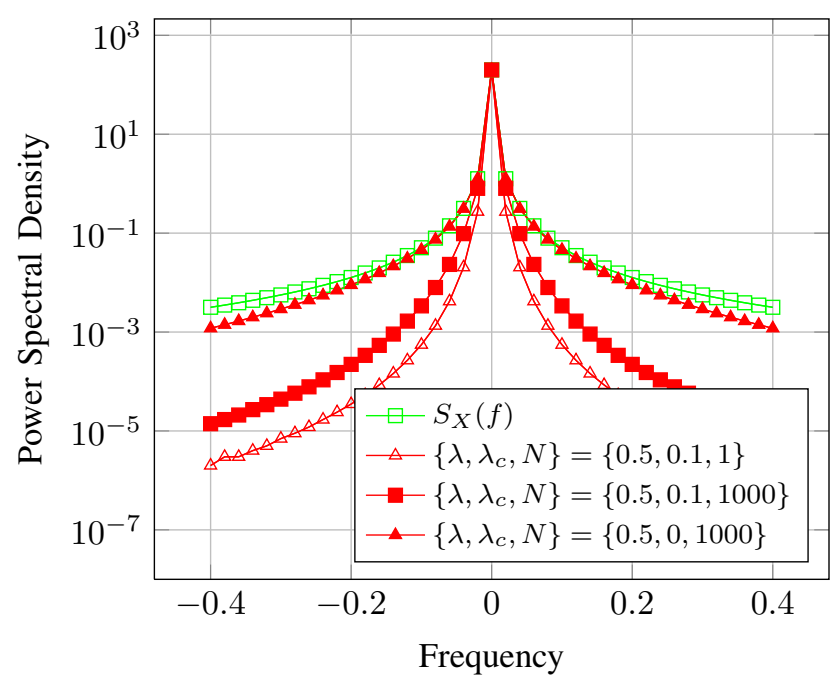

Figure 5. Comparison of the power spectra densities (PSD) of a Markov Gaussian process with different reconstructions.

have further considered that the energy harvesting processes are spatially correlated. Subsequently, in the scenario where sensors observe the same underlying phenomenon, we have analytically assessed the impact of correlated energy harvesting on the distortion. Interestingly, we have found that, for a large number of sensor nodes, the distortion tends to zero if the energy harvesting processes are independent, whereas it saturates otherwise.

\section{REFERENCES}

[1] R. J. Vullers, R. Schaijk, H. J. Visser, J. Penders, and C. V. Hoof, "Energy harvesting for autonomous wireless sensor networks," IEEE Solid-State Circuits Magazine, vol. 2, no. 2, pp. 29-38, 2010.

[2] J. Yang and S. Ulukus, "Optimal packet scheduling in an energy harvesting communication system," IEEE Trans. Wireless Commun., vol. 60, no. 1, pp. 220-230, Jan. 2012. 
[3] K. Tutuncuoglu and A. Yener, "Optimum transmission policies for battery limited energy harvesting nodes," IEEE Trans. Wireless Commun., vol. 11, no. 3, pp. 1180-1189, Mar. 2012.

[4] B. Devillers and D. Gündüz, "A General Framework for the Optimization of Energy Harvesting Communication Systems with Battery Imperfections," IEEE J. Commun. Netw., vol. 14, no. 2, pp. 130-139, 2012.

[5] O. Ozel, K. Tutuncuoglu, J. Yang, S. Ulukus, and A. Yener, "Transmission with energy harvesting nodes in fading wireless channels: Optimal policies," IEEE Trans. Wireless Commun., vol. 29, no. 8, pp. 1732-1743, Sep. 2011.

[6] O. Orhan, D. Gündüz, and E. Erkip, "Optimal packet scheduling for an energy harvesting transmitter with processing cost," in IEEE International Conference on Communications (ICC), 2013, pp. 3110 3114.

[7] W. Gardner, Introduction to random processes: with applications to signals and systems. Macmillan Pub. Co., 1986.

[8] L. Berbakov, C. Anton-Haro, and J. Matamoros, "Joint optimization of transmission policies for collaborative beamforming with energy harvesting sensors," IEEE Trans. Wireless Commun., vol. 13, no. 7, pp. 3496-3509, Jul. 2014.

[9] S. Saggini, F. Ongaro, C. Galperti, and P. Mattavelli, "Supercapacitorbased hybrid storage systems for energy harvesting in wireless sensor networks," in Twenty-Fifth Annual IEEE Applied Power Electronics Conference and Exposition (APEC),, Feb. 2010, pp. 2281-2287.

[10] D. W. Ng, E. S. Lo, and R. Schober, "Energy-efficient resource allocation in ofdma systems with hybrid energy harvesting base station," IEEE Trans. Wireless Commun., vol. 12, no. 7, pp. 3412-3427, Jul. 2013.

[11] H. S. Dhillon, Y. Li, P. Nuggehalli, Z. Pi, and J. G. Andrews, "Fundamentals of heterogeneous cellular networks with energy harvesting," IEEE Trans. Wireless Commun., vol. 13, no. 5, pp. 2782-2797, May 2014.

[12] Q. Bai and J. A. Nossek, "Modulation optimization for energy harvesting transmitters with compound poisson energy arrivals," in 2013 IEEE 14th Workshop on ignal Processing Advances in Wireless Communications (SPAWC), Jun. 2013. 